\title{
What Is a Galaxy? Cast Your Vote Here
}

\author{
Duncan A. Forbes ${ }^{\mathrm{A}, \mathrm{C}}$ and Pavel Kroupa ${ }^{\mathrm{B}}$ \\ ${ }^{\text {A }}$ Centre for Astrophysics \& Supercomputing, Swinburne University, Hawthorn VIC 3122, Australia \\ B Argelander Institute for Astronomy, University of Bonn, Auf dem Hugel 71, D-53121 Bonn, Germany \\ ${ }^{\mathrm{C}}$ Corresponding author. Email: dforbes@swin.edu.au
}

Received 2010 August 16, accepted 2010 December 27

\begin{abstract}
Although originally classified as galaxies, Ultra-Compact Dwarfs (UCDs) have many properties in common with globular star clusters. The debate on the origin and nature of UCDs, and the recently discovered ultra-faint dwarf spheroidal (dSph) galaxies which contain very few stars, has motivated us to ask the question 'What is a galaxy?' Our aim here is to promote further discussion of how to define a galaxy and, in particular, what separates it from a star cluster. Like most previous definitions, we adopt the requirement of a gravitationally bound stellar system as a minimum. In order to distinguish a dwarf galaxy from a globular cluster, we discuss other possible requirements, such as a minimum size, a long two-body relaxation time, a satellite system, the presence of complex stellar populations and non-baryonic dark matter. We briefly mention the implications of the adoption of each of these definitions. Some special cases of objects with an ambiguous nature are also discussed. Finally, we give our favoured criteria, and in the spirit of 'collective wisdom', invite readers to vote on their preferred definition of a galaxy via a dedicated website.
\end{abstract}

Keywords: galaxies: dwarf — galaxies: fundamental parameters — galaxies: star clusters — galaxies: general

\section{Introduction}

Astronomers like to classify things. That classification may initially be based on appearance to the human eye (e.g. Hubble 1926), but to make progress this taxonomy may need to have some basis in the underlying nature or physics of the objects being examined. With this mind, astronomers need a working definition so as to divide objects into different categories and to explore interesting transition cases that might share common properties. Hopefully this results in additional insight into the physical processes that are operating.

Perhaps the most famous recent case of classification in astronomy is the International Astronomical Union's definition of a planet and its separation on small scales from minor bodies in the solar system. This was partly motivated by the recent discoveries of several planet-like objects that challenged the previous loose definition of a planet. After 2 years of preparation by an IAU working group and 2 weeks of debate at an IAU General Assembly in Prague, the IAU presented its new definition of a planet. The criteria included a clause that a planet should dominate its local environment, which Pluto did not, and hence Pluto was officially stripped of its long-standing status as a planet (see also Soter et al. 2006). This decision was not uniformly welcomed, especially among the general public.

There is no widely-accepted standard definition of a galaxy. In this paper we discuss the issue of small-scale stellar systems, in particular dwarf galaxies, and what separates them from star clusters. A working definition for a dwarf galaxy was suggested in 1994 by Tammann: those galaxies fainter than $M_{B}=-16$ and more extended than globular clusters. Since that time Ultra-Compact Dwarf (UCD) objects (Hilker et al. 1999; later called galaxies by Drinkwater et al. 2000 and Phillipps et al. 2001) and ultra-faint dwarf spheroidal (dSph) galaxies around the Milky Way have been discovered. UCDs (also called Intermediate Mass Objects and Dwarf Galaxy Transition Objects) have properties intermediate between those traditionally recognised as belonging to galaxies and globular star clusters. By contrast, some of the ultrafaint $\mathrm{dSph}$ galaxies contain so few stars that they can be fainter than a single bright star and contain less stellar mass than some globular clusters (e.g. Belokrov et al. 2007).

\section{Ultra-Compact Dwarfs: Star Clusters or Galaxies?}

UCDs have sizes, luminosities and masses that are intermediate between those of objects traditionally classified as globular clusters and dwarf galaxies (e.g. Dabringhausen et al. 2008; Forbes et al. 2008; Taylor et al. 2010). Their properties and relationship with 'normal' globular clusters have been reviewed recently by Hilker (2009). Although UCDs have luminosities and stellar masses similar to those of dwarf spheroidal galaxies, they are much more compact. They have been shown to contain predominately old-aged stars and to be pressure supported (Chilingarian et al. 2010). Unlike the lower mass globular clusters (with a near constant half-light 
radius of $r_{h} \approx 3 \mathrm{pc}$ ), UCDs reveal a near linear sizeluminosity trend (Dabringhausen et al. 2008; Forbes et al. 2008; Taylor et al. 2010).

There is no universally-accepted definition of a UCD, however parameters commonly adopted are:

- $10 \leq r_{h} / \mathrm{pc} \leq 40$

- $-10.5 \geq M_{V} \geq-14$

- $2 \times 10^{6} \leq M / M_{\odot} \leq 2 \times 10^{8}$.

Some workers also apply an ellipticity criterion to ensure near-roundness (e.g. Madrid et al. 2010). We also note that UCD-like objects with half-light sizes up to $100 \mathrm{pc}$ have been reported as confirmed members of the Virgo, Fornax and Coma clusters (Evstigneeva et al. 2007; Chiboucas et al. 2010).

The issue of whether or not UCDs contain dark matter is still subject to debate, as the mass-to-light ratios are slightly higher than expected for a standard Initial Mass Function (IMF) with current stellar population models (Baumgardt \& Mieske 2008; Chilingarian et al. 2010). However, it would only take a small difference in the IMF or additional cluster evolution physics for the inferred mass-to-light ratios to be consistent with a purely stellar system devoid of dark matter (see Dabringhausen et al. 2009 for further discussion of this issue).

Formation scenarios for UCDs include a galactic origin, for example as the remnant nucleus of a stripped dwarf galaxy (Bekki et al. 2001) or as the rare surviving relic of a dwarf galaxy formed in the early universe (Drinkwater et al. 2004). The origins of star clusters may be put down to the merger of several smaller star clusters (Fellhauer \& Kroupa 2002) or simply the extension of the globular cluster sequence to higher masses (e.g. Mieske et al. 2004). Multiple origins are also possible (Chilingarian et al. 2010; Da Rocha et al. 2010; Norris \& Kannappan 2010). The observation that UCDs are consistent with the Globular Cluster (GC) luminosity (mass) function and follow a similar spatial distribution to GCs around a host galaxy would argue that most UCDs are effectively massive star clusters. So although some interesting exceptions may exist, we favour the view that UCDs today are dark matter free star clusters. Whether they should also be called 'galaxies' is discussed below.

\section{The Definition of a Galaxy}

Descriptive definitions of a galaxy are numerous. Below are three examples selected from popular websites:

A galaxy is a massive, gravitationally bound system that consists of stars and stellar remnants, an interstellar medium of gas and dust, and an important but poorly understood component tentatively dubbed dark matter.

(http: / / en.wikipedia.org/wiki /Galaxy)

Any of the numerous large groups of stars and other matter that exist in space as independent systems. (http: / / www.oed.com/)
A galaxy is a gravitationally bound entity, typically consisting of dark matter, gas, dust and stars.

(http: //astronomy.swin. edu.au/cms/astro/ cosmos/)

Most popular definitions require that a galaxy consists of matter that is gravitationally self-contained or bound. This matter could take different forms, but the presence of stars is generally required. Taking this as the starting point for the definition of a galaxy, we require that a galaxy is:

\section{Gravitationally Bound}

A fundamental criterion to be a galaxy is that the matter must be gravitationally bound (i.e. have a negative binding energy) within its own potential well. Matter that is unbound may include material stripped away by the action of a tidal encounter or 'evaporated' away if it exceeds the escape velocity of the system. If being gravitationally bound is a requirement to be a galaxy, then collections of 'tidal material' are not galaxies.

\section{Contains Stars}

An additional key requirement is that a galaxy be a stellar system, (i.e. it must include some stars). In the case of recently discovered ultra-faint dwarf spheroidal galaxies, the number of stars inferred can be as low as a few hundred.

It is possible, and indeed predicted by some simulations (e.g. Verde, Oh \& Jimenez 2002), that 'dark galaxies' exist, that is, dark matter halos containing cold gas which has for some reason failed to form any stars. In general $21 \mathrm{~cm}$ radio searches for such objects indicate that they do not exist in large numbers, if at all (Doyle et al. 2005; Kilborn et al. 2005).

If the presence of stars is a requirement to be a galaxy, then gas-rich, star-free 'dark galaxies' are not galaxies.

These two criteria taken together would exclude tidal material and 'dark galaxies', but would, however, include star clusters, such as globular clusters and UCDs, in the definition of a galaxy. Additional criteria are probably required. A few suggestions and their implications are listed below.

\section{- Two-body Relaxation Time $\geq{H_{0}^{-1}}^{-1}$}

When a stellar system is in a stable dynamical state, the orbits of the stars are determined by the mean gravity of the system rather than localised encounters between individual stars. In other words, galaxies are long-lasting systems with smooth gravitational potentials that can be modelled over time by the collisionless Boltzmann equation (Kroupa 2008). This can be quantified by calculating the two-body relaxation time (Binney \& Tremaine 1987; Kroupa 1998), i.e.

$$
t_{\text {rel }} \approx \frac{0.2}{\sqrt{G}} \frac{1}{m_{\mathrm{av}}} \frac{M^{\frac{1}{2}}}{\ln M} r^{\frac{3}{2}}
$$

where $G=0.0045 \mathrm{pc}^{3} M_{\odot}^{-1} \mathrm{Myr}^{-2}, M$ and $r$ are the mass (in $M_{\odot}$ ) and characteristic radius (in pc) of the system and 
$m_{\mathrm{av}}$ is the average stellar mass (typically $0.5 M_{\odot}$ for old stellar systems) with the relaxation time given in Myrs. Systems with a relaxation time longer than the age of the Universe would include UCDs $\left(M>10^{6} M_{\odot}\right.$ and $r>10 \mathrm{pc})$ and tidal dwarf galaxies $\left(M>10^{4} M_{\odot}\right.$ and $r>100 \mathrm{pc})$ but not star clusters traditionally classified as globular clusters $\left(M<10^{6} M_{\odot}\right.$ and $\left.r \approx 3 \mathrm{pc}\right)$.

If having a relaxation time longer than the Hubble time is a requirement to be a galaxy, then 'globular clusters' are not galaxies but 'ultra-compact dwarfs' and 'tidal dwarf galaxies' are galaxies.

\section{- Half-light Radius $\geq 100 p c$}

The half-light size, or effective radius, is a useful measure of the extent of a stellar system. As mentioned above, UCDs generally have sizes up to $40 \mathrm{pc}$. The recently discovered ultra-faint dwarf spheroidals have sizes as small as $100 \mathrm{pc}$. Thus there appears to be a zone of avoidance, which cannot be entirely due to selection effects, of size $40<r_{h}<100$ pc within which objects are very rare (Belokurov et al. 2007; Gilmore et al. 2007). Gilmore et al. have argued that the few objects within this zone of avoidance are special cases which are probably not in equilibrium but are in the throes of disruption. However the zone of avoidance is rapidly being filled, with several UCD-like objects having measured half-light sizes as large as $100 \mathrm{pc}$.

If having a half-light size greater than $\sim 100 \mathrm{pc}$ is a requirement to be a galaxy, then 'ultra-compact dwarfs' (and globular clusters) are not galaxies.

\section{- Presence of Complex Stellar Populations}

In a sufficiently deep potential well, some gas left over from the first episode of star formation will remain. This gas, and more enriched gas from stellar mass loss and supernovae, may be available for a second episode of star formation. Thus complex stellar populations of different abundances and ages will be present in substantial stellar systems. This is in contrast with the single stellar populations found in most star clusters. However, recent observational data has revealed clear evidence for multiple stellar populations in the more massive Milky Way globular clusters (Piotto 2009). A possible explanation for this is self-enrichment within a larger proto-cluster gas cloud (Parmentier 2004; Strader \& Smith 2008; Bailin \& Harris 2009). This self-enrichment process becomes apparent at masses around one million solar masses.

If the presence of complex stellar populations is a requirement to be a galaxy, then massive globular clusters (and probably 'ultra-compact dwarfs') are galaxies.

\section{- Presence of Non-baryonic Dark Matter}

Our standard paradigm of galaxy formation is that every galaxy formed in a massive dark matter halo (White \& Rees 1978). Thus the presence of dark matter is seen by many as a key requirement to be classified as a galaxy (e.g. Gilmore et al. 2007). It is unfortunately a difficult property to measure empirically for dwarf galaxies, usually relying on measurements of the velocity dispersion. So although high dark matter fractions have been inferred for Local Group dSph galaxies (assuming the velocity dispersion is a valid diagnostic for these systems), dE galaxies indicate very little dark matter within the half-light radius (Forbes et al. 2010; Toloba et al. 2010) and perhaps to $\sim 10$ times the half-light radius in the case of NGC 147 and NGC 185, depending on the choice of IMF and stellar population model (Geha et al. 2010). An alternative explanation to the measured high velocity dispersions is that non-Newtonian dynamics are operating (Brada \& Milgrom 2000; McGaugh \& Wolf 2010).

We note that tidal dwarf galaxies, that form out of the collapse of disk material in a tidal tail after a merger, are not expected to contain much dark matter (Barnes \& Hernquist 1992; Gentile et al. 2007). Thus if any of the dSph galaxies which surround the Milky Way in a disk of satellites (Metz et al. 2009; Kroupa et al. 2010) have a tidal dwarf origin they would not be expected to have a high dark matter content (and the observed velocity dispersions used to infer the presence of dark matter would be an invalid diagnostic).

The dark matter galaxy formation scenario may extend down to GCs which have been suggested to form in (mini) dark matter halos (Bromm \& Clarke 2002; Mashchenko \& Sills 2005; Saitoh et al. 2006; Griffen et al. 2010). However, they must have lost this dark matter as none as been detected to date in Milky Way GCs (Moore 1996; Lane et al. 2010; Conroy, Loeb \& Spergel 2010).

If the presence of a massive dark matter halo is a requirement to be a galaxy, then probably 'tidal dwarf galaxies', 'ultra-compact dwarfs', and possibly some Milky Way 'dwarf spheroidal galaxies' and 'dwarf elliptical galaxies' are not galaxies.

\section{- Hosts a Satellite Stellar System}

Evidence that a galaxy dominates its environment could come from the presence of smaller satellite stellar systems, such as dwarf galaxies (for large galaxies) or globular clusters. All known large galaxies possess a system of globular clusters, however some dwarf galaxies do not host any globular clusters (e.g. Forbes 2005). For example, in the Local Group, the dwarf galaxy WLM has a single globular cluster but the galaxies Aquarius, Tucana and the recently discovered ultra-faint dwarf satellites of the Milky Way appear to have none.

If the presence of a globular cluster system is a requirement to be a galaxy, then 'ultra-compact dwarfs' and some of the smallest 'dwarf galaxies' are not galaxies.

Of course we don't live in a static Universe, and an object could evolve from a galaxy into a star cluster (or vice versa). For example, it has been suggested that globular clusters may sink to the centre of a galaxy via dynamical friction, forming a galaxy nucleus. If that galaxy then loses its outer stars from tidal stripping, leaving only the remnant nucleus, it may be classified as a UCD or a globular cluster. Passive evolution or 
interactions (mergers, tidal stripping, etc.) can change the nature of an object over time. The criteria listed above apply to objects today and not their past or future state.

Below we briefly mention some special cases of stellar systems which challenge attempts to define a galaxy.

\section{Special Cases}

\section{- Omega Cen and G1}

Omega Cen has traditionally been known as the most massive globular cluster in the Milky Way system. However, the presence of multiple stellar populations, its large size, elongation, helium abundance and retrograde orbit have led many to suggest that it is actually the remnant nucleus of a disrupted dwarf galaxy (Freeman 1993; Bekki \& Freeman 2003). It may therefore represent a (low-mass) example of a UCD. Similar arguments have been made for the globular cluster G1 in M31 (Meylan et al. 2001; Bekki \& Chiba 2004). Otherwise, both Omega Cen and G1 are consistent with the general scaling properties of massive globular clusters (e.g. Forbes et al. 2008).

\section{- Willman 1, Segue 1, Segue 2 and Bootes II}

Willman 1 (Willman et al. 2005), Segue 1 (Belokurov et al. 2007), Segue 2 (Belokurov et al. 2009) and Bootes II (Walsh, Jerjen \& Willman 2007) are all low surfacebrightness objects discovered recently in deep surveys. They have low luminosities of $M_{V} \sim-2$ (stellar masses of a few hundred solar masses) and half-light sizes of $r_{h} \sim$ $30 \mathrm{pc}$. Such values place them at the extreme of the globular cluster distribution, with relaxation timescales much shorter than the age of the Universe. In the case of Segue 1, Geha et al. (2009) suggest that it is a galaxy with a mass-to-light ratio of $\sim 1200$ on the basis of a measured velocity dispersion of $4.2 \pm 1.2 \mathrm{~km} \mathrm{~s}^{-1}$. Subsequently, Niederste-Ostholt et al. (2009) found that this velocity dispersion may be inflated by nearby Sagittarius dwarf galaxy stars, and favoured a globular cluster status for Segue 1. Most recently, Simon et al. (2010) have reiterated that Seque 1 is a dark-matter dominated dwarf galaxy. They conclude that stars from the Sagittarius dwarf do not unduly affect their results and that 'the metallicities of stars in Segue 1 provide compelling evidence that, irrespective of its current dynamical state, Segue 1 was once a dwarf galaxy'.

\section{- Coma Berenices}

Coma Berenices was discovered by Belokurov et al. (2007). Deep imaging by Munoz, Geha \& Willam (2010) indicates a half-light size of $r_{h}=74 \mathrm{pc}$ and an ellipticity of 0.36 . The $\mathrm{V}$-band luminosity was determined to be $M_{V}=-3.8$. Thus it has a similar luminosity to Willman 1, Segue 1, 2 and Bootes II but is significantly larger in size. Its size places it within the half-light zone of avoidance between the locus of globular clusters/UCDs and dwarf galaxies. However, there is no obvious sign of tidal stripping to faint surface-brightness levels. Simon \&
Geha (2007) derive a metallicity $[\mathrm{Fe} / \mathrm{H}]=-2$ with zero dispersion. However, Kirby et al. (2008) quote a mean metallicity of $[\mathrm{Fe} / \mathrm{H}]=-2.53$ with a large dispersion of 0.45 dex. The latter suggests that multiple stellar populations may be present in Coma Berenices. We note that the Simon \& Geha (2007) metallicity for Coma Berenices is similar to that for GCs of a comparable luminosity, whereas the Kirby et al. (2008) metallicity is more metalpoor than the most metal-poor Milky Way GC and is consistent with an extrapolation of the metallicity-luminosity relation to lower stellar masses.

\section{- VUCD7 and F-19}

The UCDs VUCD7 in the Virgo cluster (Evstigneeva et al. 2007) and F-19 in the Fornax cluster (also known as UCD3; Mieske et al. 2008) are classified as very luminous UCDs with $M_{V} \sim-13.5$, measured sizes of $r_{h} \sim 90 \mathrm{pc}$ and central velocity dispersions of $\sigma \sim 25 \mathrm{~km} \mathrm{~s}^{-1}$. These values imply masses of $\sim 10^{8} M_{\odot}$ and a location within the half-light zone of avoidance. However, both of these objects might be better described as UCDs with an extended $(\sim 200 \mathrm{pc})$ envelope of stars. They may represent transition objects between nucleated dwarfs and (envelope-free) UCDs.

\section{- $\mathrm{M} 59 \mathrm{cO}$}

M59cO (also known as SDSS J124155.33+114003.7), located in the Virgo cluster, was discovered by Chilingarian \& Mamon (2008). They measured its key properties to be $r_{h}=32 \mathrm{pc}, \sigma=48 \mathrm{~km} \mathrm{~s}^{-1}$ and $M_{V} \sim$ -13.5 , and suggested that it is a transition object between UCDs and compact ellipticals like M32. However, its properties are much closer to those of UCDs than M32.

\section{- NGC 4546 UCD1}

Norris \& Kannappan (2010) report the discovery of a UCD with $M_{V} \sim-13$ associated with the nearby S0 galaxy NGC 4546. This UCD is found to have a young age of $\sim 3 \mathrm{Gyr}$ and to be counter-rotating with respect to the stars in NGC 4546 (although, interestingly, it corotates with the gas around NGC 4546). The high luminosity, young age and retrograde orbit of the NGC 4546 UCD would make it a prime candidate for a stellar system that is not simply a massive globular cluster of NGC 4546, but rather an object that was formed, or accreted, in a tidal interaction some 3 Gyrs ago.

\section{- Bootes III, Hercules and Ursa Major II}

These may be objects in transition between a bound dwarf galaxy and unbound tidal material. In the case of Bootes III, which is on a highly radial orbit, Carlin et al. (2009) argue that its internal kinematics and structure suggest an object in the process of tidal disruption. It shows evidence for a metallicity spread in its stars. Hercules is perhaps the most elongated Milky Way dSph galaxy (apart from the disrupted Sagittarius dwarf galaxy) with an ellipticity from deep imaging of $\sim 0.65$ and a 
Table 1. Are these stellar systems galaxies?

\begin{tabular}{lccccc}
\hline Object & $t_{\text {relax }} H_{0}^{-1}$ & $r_{h}>100 \mathrm{pc}$ & Multi-pops & Dark matter & Satellites \\
\hline Omega Cen & $\times$ & $\times$ & $\sqrt{ }$ & $\times$ & $\times$ \\
Segue 1 & $\times$ & $\times$ & $+\sqrt{ }^{\mathrm{a}}$ & $\times$ \\
ComBer & $\sqrt{ }$ & $\times$ & $?$ & $\sqrt{ }{ }^{\mathrm{a}}$ & $?$ \\
VUCD7 & $\sqrt{ }$ & $\times$ & $?$ & $?$ & $\times$ \\
M59cO & $\sqrt{ }$ & $\sqrt{ }$ & $\sqrt{ }$ & $?$ & $\times$ \\
BooIII & $\sqrt{ }$ & $\sqrt{ }$ & $\times$ & $\times$ \\
VCC2062 & $\sqrt{ }$ & & $?$ & $\times$ \\
\hline
\end{tabular}

${ }^{a}$ Presence of dark matter assumes that the observed velocity dispersion is a valid diagnostic and not due to other effects (e.g. interlopers, binary stars, tidal heating, non-Newtonian dynamics, etc.).

half-light size of $\sim 170 \mathrm{pc}$ (Coleman et al. 2007). We note that its elongation (and other properties) resemble model RS1-5 of Kroupa (1997). This simulation followed the tidal disruption of a dwarf galaxy, in a Milky-Way — like halo, that formed without dark matter (e.g. from condensed gas in a tidal tail). Ursa Major II shows signs of ongoing tidal interaction (Munoz et al. 2010). Both Hercules and Ursa Major II reveal evidence for multiple stellar populations (Kirby et al. 2008).

\section{- $V C C 2062$}

Duc et al. (2007) have suggested that VCC 2062, located in the Virgo cluster, is a tidal dwarf galaxy formed as the result of an interaction involving NGC 4694 and another galaxy. It contains a large quantity of cold gas and exhibits low-level ongoing star formation, along with evidence of older $(0.3 \mathrm{Gyr})$ stars. It has a total luminosity of $M_{B}=-13$ and size of a few kpc. The HI gas reveals a velocity gradient indicative of rotation. The baryonic (i.e. stellar and cold gas) mass content accounts for a large fraction of the inferred dynamical mass of VCC 2062.

A summary of how some special-case objects match up to the different criteria given above is given in Table 1 . If the object satisfies the requirement to be a galaxy it is assigned a ' $\sqrt{ }$ ', a ' $x$ ' if it fails and a '?' if it is currently uncertain. As far as we are aware none of the special case objects hosts a satellite, thus each is assigned a ' $x$ ' in that column of Table 1. The presence of dark matter is often controversial and assumes that the measured velocity dispersion is not dominated by interlopers, binary stars or tidal heating effects (the latter is questionable for Bootes III). Under this assumption, two of the objects have good evidence for a high non-baryonic dark matter content. Even if we exclude the satellites criterion, none of the objects listed in Table 1 satisfies all of the criteria.

\section{Conclusions}

Here we have accepted the popular definition of a galaxy requiring that it be both gravitationally bound and consist of a system of stars. As such criteria would include globular (star) clusters, additional criteria are required to define a galaxy. We suggest that the next best criterion is a dynamical one, i.e. that the stars are collisionless, subject to the general gravitational field of the system. This can be usefully quantified using the two-body relaxation time. With these three criteria, globular clusters are effectively excluded from the definition of a galaxy, as are Omega Cen, Segue 1 (and similar objects) and Coma Berenices. However Ultra-Compact Dwarfs (and perhaps the most massive globular clusters) would be classed as galaxies. Although this may satisfy some, a fourth criterion would be required to exclude Ultra-Compact Dwarfs. We suggest a size-based criterion, e.g. half-light radius greater than $100 \mathrm{pc}$. This fourth criterion would exclude the vast bulk of known Ultra-Compact Dwarfs but may still include extreme objects such as VUCD7 and F-19. Bootes III (and similar objects, assuming they are gravitationally bound) and tidal dwarfs like VCC 2062 would also be classed as galaxies.

The combination of criteria above is somewhat subjective and the opinion of two astronomers. The decision of how to define a small planet, and hence the taxonomic fate of Pluto, was decided by 424 astronomers present on the last day of the IAUGeneral Assembly in Prague, held in August 2006. In order to capture the thoughts of a wider audience about how to define a galaxy, we invite readers to vote. This 'collective wisdom' or 'crowd-sourcing' will be captured in an online poll. The poll allows one to choose the single best criterion or multiple criteria. Results of the poll will be reported from time to time at future astronomy conferences. The website for anonymous voting is: http://www.surveymonkey. com/s / WLRJMWS.

\section{Acknowledgments}

We thank the following for useful discussions: C. Foster, A. Graham, D. Koo, E. Ryan-Weber, J. Strader.

\section{References}

Bailin, J. \& Harris, W., 2009, ApJ, 695, 1082

Barnes, J. \& Hernquist, L., 1992, Nature, 360, 715

Baumgardt, H. \& Mieske, S., 2008, MNRAS, 391, 942

Bekki, K., Couch, W. \& Drinkwater, M., 2001, ApJ, 552, 105

Bekki, K. \& Freeman, K., 2003, MNRAS, 346, 11

Bekki, K. \& Chiba, M., 2004, A\&A, 417, 437

Belokurov, V. et al., 2007, ApJ, 654, 897

Belokurov, V. et al., 2009, MNRAS, 397, 1748

Binney, J. \& Treamine, S., 1987, Galactic Dynamics, Princeton University Press

Brada, R. \& Milgrom, M., 2000, ApJ, 541, 556 
Bromm, V. \& Clarke, C., 2002, ApJ, 566, L1

Carlin, J. et al., 2009, ApJ, 702, 9

Chiboucas, K. et al., 2010, arXiv:1009.3950

Chilingarian, I. \& Mamon, G., 2008, MNRAS, 385, 83

Chilingarian, I., Mieske, M., Hilker, M. \& Infante, L., MNRAS, submitted

Coleman, M. et al., 2007, ApJ, 668, L43

Conroy, C., Loeb, A. \& Spergel, D., 2010, ApJ, in press

Dabringhausen, J., Hilker, M. \& Kroupa, P., 2008, MNRAS, 386,864

Dabringhausen, J., Kroupa, P. \& Baumgardt, H., 2009, MNRAS, 394,1529

Da Rocha, C., Mieske, S., Georgiev, I. Y., Hilker, M., Ziegler, B. L. \& Mendes de Oliveira, C., arXiv: 1009.3010

Diemand, J. et al., 2008, Nature, 454, 735

Doyle, M. et al., 2005, MNRAS, 361, 34

Drinkwater, M. et al., 2000, PASA, 17, 227

Drinkwater, M. et al., 2004, PASA, 21, 375

Duc, P.-A. et al., 2007, A\&A, 475, 187

Evstigneeva, E., Gregg, M., Drinkwater, M. \& Hilker, M., 2007, AJ, 133,1722

Fellhauer, M. \& Kroupa, P., 2002, MNRAS, 330, 642

Forbes, D., 2005, ApJ, 635, 137

Forbes, D. A., Lasky, P., Graham, A. W. \& Spitler, L., 2008, MNRAS, 389, 1924

Forbes, D., Spitler, L., Graham, A., Foster, C., Hau, G. \& Benson, A., 2010, MNRAS, submitted

Freeman, K., 1993, ASPC, 48, 608

Geha, M. et al., 2009, ApJ, 692, 1464

Geha, M. et al., 2010, ApJ, 711, 361

Gentile, G., Famaey, B., Combes, F., Kroupa, P., Zhao, H. S. \& Tiret, O., 2007, A\&A, 472, L25

Gilmore, G. et al., 2007, ApJ, 663, 948

Griffen, B. et al., 2010, MNRAS, 405, 375

Hilker, M. et al., 1999, A\&A, 134, 75

Hilker, M., 2009, ariX:0906.0776

Hubble, E., 1926, ApJ, 64, 321

Kilborn, V. et al., 2005, PASA, 22, 326

Kirby, E., Simon, J., Geha, M., Guhathakurta, P. \& Frebel, A., 2008, ApJ, 685, 43L
Kroupa, P., 1997, New Astronomy, 2, 139

Kroupa, P., 1998, MNRAS, 300, 200

Kroupa, P., 2008, in Dynamical Evolution of Dense Stellar Systems, IAU Symposium 246, 13

Kroupa, P. et al., 2010, A\&A, 523, 32

Lane, R. et al., 2010, MNRAS, 406, 2732

Madrid, J. et al., 2010, MNRAS, submitted

Mashchenko, S. \& Sills, A., 2005, ApJ, 619, 258

McGaugh, S. \& Wolf, J., 2010, arXiv:1003.3448

Meylan, G., Sarajedini, A., Jablonka, P., Djorgovski, S. G., Bridges, T. \& Rich, R. M., 2001, AJ, 122, 830

Metz, M., Kroupa, P., Theis, C., Hensler, G. \& Jerjen, H., 2009, ApJ, 697, 269

Mieske, S., Hilker, M. \& Infante, L., 2004, A\&A, 418, 445

Mieske, S. et al., 2008, A\&A, 487, 921

Moore, B., 1996, ApJ, 461, L13

Munoz, R., Geha, M. \& Willman, B., 2010, AJ, 140, 138

Niederste-Ostholt, M., Belokurov, V., Evans, N., Gilmore, G., Wyse, R. \& Norris, J., 2009, MNRAS, 398, 1771

Norris, M. \& Kannappan, S., arXiv:1009.2489

Parmentier, G., 2004, MNRAS, 351, 585

Phillipps, S., Drinkwater, M. J., Gregg, M. D. \& Jones, J. B., 2001, ApJ, 560, 201

Piotto, G., 2009, arXiv:0902.1422

Saitoh, T., Koda, J., Okamoto, T., Wada, K. \& Habe, A., 2006, ApJ, 640,22

Simon, J. \& Geha, M., 2007, ApJ, 670, 313

Simon, J. et al., 2010, arXiv:1007.4198

Soter, S., 2006, AJ, 132, 2513

Strader, J. \& Smith, G., 2008, AJ, 136, 1828

Tammann, G., 1994, ESOC, 49, 3

Taylor, M. A., Puzia, T. H., Harris, G. L., Harris, W. E., KisslerPatig, M. \& Hilker, M., 2010, ApJ, 712, 1191

Toloba, E. et al., 2010, A\&A, submitted

Verde, L., Oh, S. \& Jimenez, R., 2002, MNRAS, 336, 541

Walsh, S. M., Jerjen, H. \& Willman, B., 2007, ApJ, 662, L83

White, S. \& Rees, M., 1978, MNRAS, 183, 341

Willman, B. et al., 2005, ApJ, 626, 85 